Provided for non-commercial research and education use. Not for reproduction, distribution or commercial use.

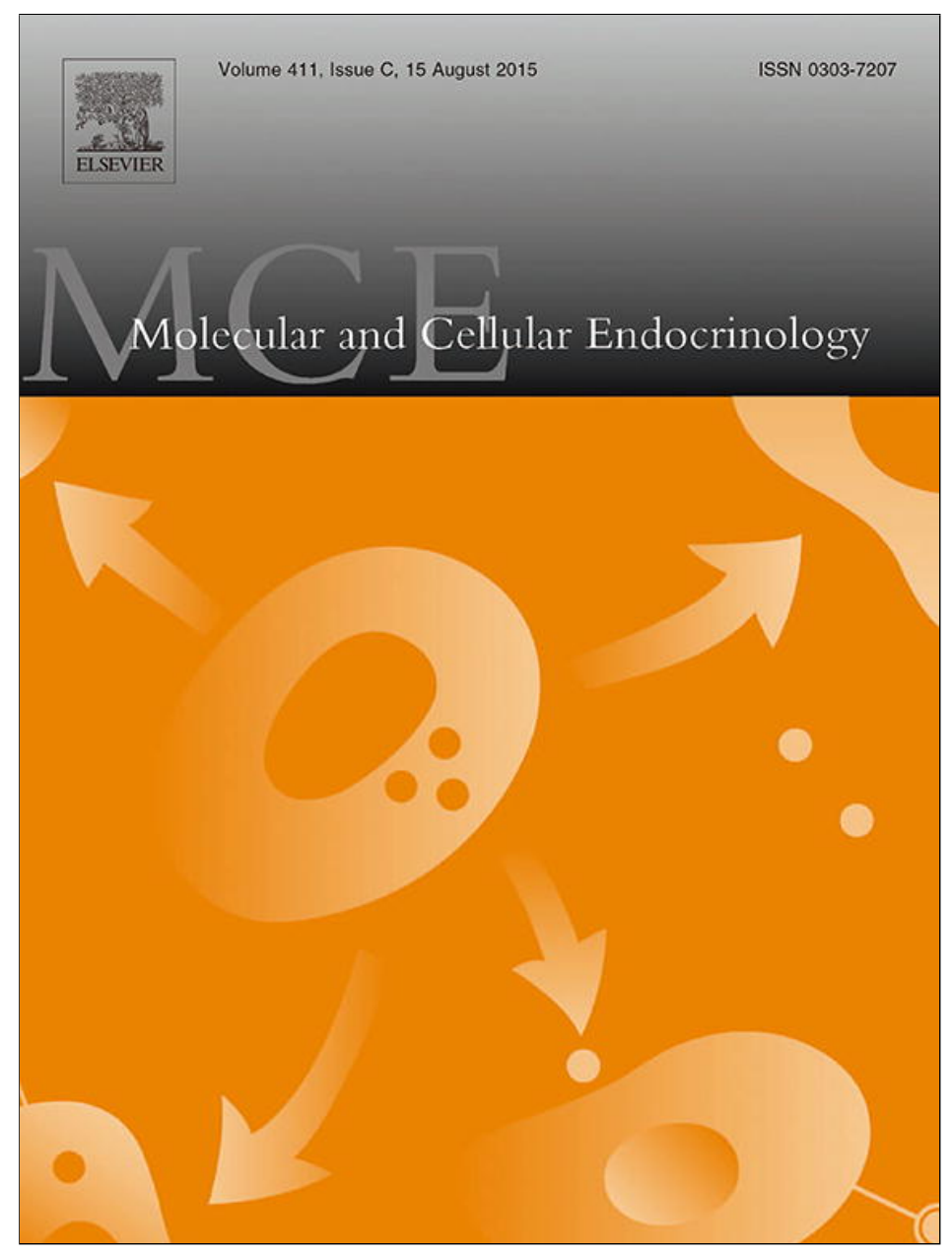

This article appeared in a journal published by Elsevier. The attached copy is furnished to the author for internal non-commercial research and education use, including for instruction at the authors institution and sharing with colleagues.

Other uses, including reproduction and distribution, or selling or licensing copies, or posting to personal, institutional or third party websites are prohibited.

In most cases authors are permitted to post their version of the article (e.g. in Word or Tex form) to their personal website or institutional repository. Authors requiring further information regarding Elsevier's archiving and manuscript policies are encouraged to visit:

http://www.elsevier.com/authorsrights 


\title{
Immunohistochemical, genetic and clinical characterization of sporadic aldosterone-producing adenomas
}

\author{
Silvia Monticone ${ }^{\mathrm{a}, *, 1}$, Isabella Castellano ${ }^{\mathrm{b}, 1}$, Karine Versace ${ }^{\mathrm{d}}$, Barbara Lucatello ${ }^{\mathrm{c}}$,

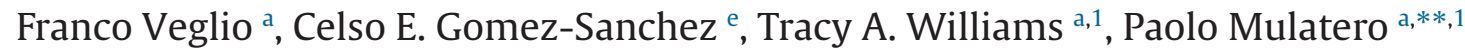 \\ a Division of Internal Medicine and Hypertension, Department of Medical Sciences, University of Torino, Torino, Italy \\ b Division of Pathology, Department of Medical Sciences, University of Torino, Torino, Italy \\ ${ }^{c}$ Division of Endocrinology, Department of Medical Sciences, University of Torino, Torino, Italy \\ d Division of Radiology, University of Torino, Torino, Italy \\ e Division of Endocrinology, G.V. (Sonny) Montgomery VA Medical Center and University of Mississippi Medical Center, Jackson, MS 39216, USA
}

\section{A R T I C L E I N F O}

\section{Article history:}

Received 12 January 2015

Received in revised form 25 April 2015

Accepted 26 April 2015

Available online 6 May 2015

\section{Keywords:}

Aldosterone producing adenoma

CYP11B1 immunohistochemistry

CYP11B2 immunohistochemistry

KCNJ5

ATP1A1

CACNA1D

\begin{abstract}
A B S T R A C T
Adrenal glands removed for unilateral primary aldosteronism (PA) display marked histological heterogeneity. Recently reported somatic mutations in KCNJ5, ATP1A1, ATP2B3 and CACNA1D can partially account for these differences. In this study we aimed at combining phenotypic and genotypic characteristics, integrating genetic and immunohistochemistry correlates in sporadic PA. Seventy-one adrenal glands have been included in the study and analyzed for mutations in KCNJ5, ATP1A1, ATP2B3 and CACNA1D. Histological examination and immunohistochemical staining for CYP11B1 (11ß-hydroxylase) and CYP11B2 (aldosterone synthase) were performed on aldosterone-producing adenomas (APAs) and adjacent adrenal cortex. In our cohort, the final histopathological diagnosis was multinodular hyperplasia in $22.5 \%$ of the patients and single nodule in 77.5\%. Forty-five percent of the removed adrenals displayed extra-APA CYP11B2-positive cell nests (B2-CN). Among adrenal vein sampling parameters the suppression of contralateral adrenal was more frequent and the lateralization index was higher in the subgroup of patients without extra-APA B2-CN compared to the subgroup with extra-APA B2-CN. KCNJ5-mutated APAs were composed mainly of zona fasciculata-like cells with high expression of CYP11B1, while ATP1A1, ATP2B3 and CACNA1D-mutated APAs presented more frequently a zona-glomerulosa-like phenotype with high expression of CYP11B2. We observed a significant inverse correlation between CYP11B2 expression and the size of the nodules and, if CYP11B2 expression was corrected for tumor volume, a significant correlation with plasma aldosterone and aldosterone to renin ratio. Our findings indicate that combination of genotyping and immunohistochemistry improves the final histopathological diagnosis between single nodule and multinodular hyperplasia of the assessed adrenals.
\end{abstract}

(C) 2015 Elsevier Ireland Ltd. All rights reserved.

\section{Introduction}

Primary aldosteronism (PA) is the most frequent cause of endocrine hypertension (Funder et al., 2008) and affected patients display an increased risk of cardio- and cerebro-vascular

Abbreviations: PA, primary aldosteronism; APA, aldosterone producing adenoma; $\mathrm{BAH}$, bilateral adrenal hyperplasia; AVS, adrenal vein sampling.

* Corresponding author. Department of Medical Sciences, Division of Internal Medicine and Hypertension, University of Torino, Via Genova 3, 10126 Torino, Italy. Tel.: +39 0116336959; fax: +390116336931.

E-mail address: smv.monticone@gmail.com (S. Monticone).

** Corresponding author. Department of Medical Sciences, Division of Internal Medicine and Hypertension, University of Torino, Via Genova 3, 10126 Torino, Italy. Tel.: +39 0116336959; fax: +390116336931.

E-mail address: paolo.mulatero@libero.it (P. Mulatero).

1 Equal contributions. complications compared with age-, sex- and BP-matched essential hypertensives (Mulatero et al., 2013). Aldosterone producing adenoma (APA) and bilateral adrenal hyperplasia (BAH) account for the great majority of all PA cases (Mulatero et al., 2004). The differential diagnosis between APA and BAH is of fundamental importance to allocate affected patients to appropriate management: either unilateral adrenalectomy for an APA or medical therapy with mineralocorticoid receptor antagonists for BAH. Preoperative subtype diagnosis is based on computed tomography (CT) scanning and adrenal vein sampling (AVS), which is the only reliable approach to distinguish between unilateral and bilateral disease (Funder et al., 2008). CT scanning has been widely demonstrated to lack sensitivity and specificity since micro-APAs (less than $1 \mathrm{~cm}$ in diameter) are not always detectable and a non-secreting nodule in the context of a bilateral hyperplasia may be misclassified as an APA (Funder et al., 2008; Mulatero et al., 2008). Preoperative diagnosis of APA is usually confirmed by the cure or significant amelioration of hypertension, 
normalization of hypokalemia and aldosterone-renin ratio (ARR) and also by the histological examination of the adrenal that frequently reveals macro- and microscopic heterogeneities. Most adrenals with an APA comprise a single well-circumscribed nodule, although frequently the removed gland displays macro- or micronodular hyperplasia (Dekkers et al., 2014), with various degrees of adrenal cortex remodeling, that is atrophic, with diffuse hyperplasia or nodular hyperplasia (Boulkroun et al., 2010).

In the adult adrenal gland the exclusive site of aldosterone production is the zona glomerulosa, the localization of CYP11B2 gene expression that encodes the rate-limiting enzyme of aldosterone production, aldosterone synthase. However, the most common APAs are composed mainly of zona fasciculata (ZF)-like cells (large clear cells with lipid-laden cytoplasm and small nuclei). Less frequent are APA comprising zona glomerulosa (ZG)-like cells (small, with a high nuclear-cytoplasmic ratio and a smaller lipid content compared to ZF-like cells) or hybrid cells (intermediate between ZF-like and ZGlike cells) (Neville and O'Hare, 1985). Histopathological differences in cellular composition have been proposed to account for the different aldosterone responses to angiotensin II (AII) in APAs (Tunny et al., 1991): tumors predominantly fasciculata-like $(\geq 50 \%)$ have been reported to be mostly AII-unresponsive (AII-U), in contrast, AIIresponsive (AII-R) APAs contain less than $20 \%$ of ZF-like cells (Tunny et al., 1991). Immunohistochemical analyses of the steroidogenic enzymes aldosterone synthase (CYP11B2) and 11 -hydroxylase (CYP11B1, which catalyzes the terminal step of cortisol synthesis) provide important functional information and help the histopathological diagnosis of PA. Adrenal nodules and the adjacent adrenal cortex display varied distributions of $11 \beta$-hydroxylase and various degrees of aldosterone synthase expression, including cell clusters that exhibit strong CYP11B2 immunostaining despite the suppressed renin-angiotensin system, called aldosterone producing cell clusters (APCCs), whose functional significance has not been fully elucidated (Dekkers et al., 2014; Nishimoto et al., 2010).

Identification of somatic mutations in genes involved in $\mathrm{Ca}^{2+}$ homeostasis (KCNJ5, ATP1A1, ATP2B3 and CACNA1D) in almost 55$70 \%$ of sporadic APAs has represented a major advance in our understanding of the pathogenesis of PA (Monticone et al., 2014a). Clinical and biochemical correlates of somatic mutations in APAs revealed that KCNJ5 mutations were significantly more prevalent in females than in males and in young patients (Boulkroun et al., 2012; Fernandes-Rosa et al., 2014) with large adenomas (Azizan et al., 2013), whereas CACNA1D mutations were frequently identified in small adenomas (Fernandes-Rosa et al., 2014).

In this study we exploited the recently developed monoclonal antibodies against $11 \beta$-hydroxylase and aldosterone synthase (Gomez-Sanchez et al., 2014) to integrate clinical, immunohistochemical and genetic correlates in a cohort of 71 adrenal glands removed following the diagnosis of unilateral PA.

\section{Materials and methods}

\subsection{Patients selection}

Adrenal glands included in the study were removed from patients affected by unilateral PA, diagnosed in two tertiary referral hypertension centers (Division of Internal Medicine 4 - Hypertension Unit and Division of Endocrinology), at the University of Torino, Italy. Case detection and subtype differentiation were performed according to the Endocrine Society Guidelines (Funder et al., 2008) as described previously (Monticone et al., 2014b). Briefly, after withdrawal of interfering medications, aldosterone to plasma renin activity ratio (ARR) was used as a screening test for PA. The diagnosis was confirmed with i.v. saline loading test or captopril challenge test (when acute plasma volume expansion was contraindicated) and patients with confirmed PA underwent adrenal CT scanning and adrenal vein sampling (AVS). Two patients underwent adrenalectomy without AVS: in one case adrenal CT scanning revealed a single nodule greater than $4 \mathrm{~cm}$, suspicious for adrenocortical carcinoma and in the other case the patients refused the procedure and was considered to have "high probability of APA" (Young et al., 2004). In two cases AVS was not successful and the patients underwent unilateral adrenalectomy on the basis of positive adrenal CT findings and clinical parameters. AVS procedures were performed as described previously (Monticone et al., 2012); AVS was considered to be successful if the cortisol adrenal vein $_{\text {/cortisol }}$ peripheral vein was $>3$ ( $>2$ before 2008) and to show lateralization when the aldosterone/cortisol adrenal vein/ $_{1}$ aldosterone/cortisol contralateral adrenal vein $_{\text {was }}>4$ or if it was $>3$ together with contralateral suppression (aldosterone/cortisol in the contralateral vein lower than that in the peripheral vein). All patients showing lateralization at the AVS underwent unilateral laparoscopic adrenalectomy. Resected adrenals were assessed macroscopically and microscopically. The diagnosis of unilateral PA was confirmed by cure or significant amelioration of hypertension, normokalemia, normal ARR and low aldosterone levels and/or the normal suppressibility of aldosterone (follow up data available for $69 / 71$ patients). The posture test (available for 51 patients) was performed by measuring plasma aldosterone at 08:00 h after recumbency and after $2 \mathrm{~h}$ of standing; a greater than $50 \%$ increase in plasma aldosterone levels over basal was considered a positive test result. All patients displaying at adrenal CT scanning a nodule greater than $1 \mathrm{~cm}$ underwent $1 \mathrm{mg}$-overnight dexamethasone suppression test to exclude subclinical hypercortisolism. The test was considered positive when plasma cortisol failed to suppress to less than $50 \mathrm{nmol} / \mathrm{L}$ (Fallo et al., 2011). All patients gave their written informed consent for the use of the samples and the clinical data and the protocol of the study was approved by our local ethics committee.

\subsection{DNA sequencing of KCNJ5, ATP1A1, ATP2B3 and CACNA1D}

DNA fragments from KCNJ5, ATP1A1, ATP2B3 and CACNA1D were PCR amplified using primers reported previously (Fernandes-Rosa et al., 2014; Williams et al., 2014). The validity of novel mutations was confirmed by sequencing both strands of an independently amplified PCR fragment. The present cohort of 71 APA samples ( 55 from the Division of Internal Medicine and Hypertension and 16 from the Division of Endocrinology) were all included in the study by Fernandes-Rosa et al. (2014). Hot spot regions for mutations in KCNJ5, ATP1A1, ATP2B3 and CACNA1D were sequenced in all 71 APAs included in the study. The overall prevalence of somatic mutations was $51 \%$ (36/71) with $38 \%$ (27/71) of KCNJ5 mutations, 8.5\% (6/71) of ATPase mutations (5 ATP1A1 mutations and 1 ATP2B3 mutation) and $4.2 \%$ (3/71) of CACNA1D mutations. Clinical and biochemical data of the patients included in the study, before and after adrenalectomy, according to the mutational status are summarized in Supplementary Table S1.

\subsection{Pathological analysis}

Histological examination was performed by an experienced pathologist (I.C.) on APAs and adjacent adrenal cortex. All adrenal glands included in the study were paraffin embedded, cut into $3 \mu \mathrm{m}$ thick slices and stained with H\&E. The diagnosis of single nodule was considered when only one tumor was present; the diagnosis of multinodular hyperplasia was considered when several nodules of different sizes could be seen (with or without a dominant nodule). Cellular composition was determined by examining for known features of zona fasciculata (large, lipid-laden clear cells, with round to oval vesicular nuclei), zona glomerulosa (small, compact cells, with high nuclear/cytoplasmic ratio and moderate amount of lipid) and zona reticularis (lipid-sparse cytoplasm, compact cells) cells (Nakamura et al., 2014; Neville and O'Hare, 1985). The tumors were 
classified as ZF-like when the percentage of large vacuolated cells was greater than $50 \%$, and ZG-type when the percentage of ZFlike cells was $<50 \%$ and ZG-type cells were the most prominent cell type. The adrenal cortex adjacent to a nodule was examined for the presence of extra-APA CYP11B2-positive cell nest (B2-CN) (in two samples no adjacent adrenal cortex was available for microscopic examination), defined as cell clusters showing CYP11B2 but not CYP11B1 immunohistochemical expression (Dekkers et al., 2014; Nishimoto et al., 2010). Assuming the tumor shape as a sphere, the tumor volume was estimated using the formula: volume $=4 / 3 \pi r^{3}$, where " $r$ " represents the radius of the nodule measured by microscopical evaluation.

\subsection{Immunohistochemical and immunofluorescence procedure}

Immunohistochemistry was performed using the following primary antibodies: rat monoclonal anti-human CYP11B1-80-7 (11ßhydroxylase) and mouse monoclonal anti-human CYP11B2-41-17 (aldosterone synthase) (Gomez-Sanchez et al., 2014). For both protocols, sections of $3 \mu \mathrm{m}$ thickness from paraffin-embedded adrenal tissue were incubated with $\mathrm{H}_{2} \mathrm{O}_{2}$, and pre-treated with EDTA $0.1 \mathrm{mM}$ (pH 8) for 40 minutes at $98{ }^{\circ} \mathrm{C}$ for antigen retrieval. Subsequently, to detect CYP11B1 expression, after endogenous biotin blocking by sequential avidin-biotin treatment, the slides were incubated overnight at $4{ }^{\circ} \mathrm{C}$ with the primary antibody diluted $1: 100$. After rinsing in PBS, slides were treated with biotinylated secondary antibody goat anti-rat (STARD131, AbD Serotec, diluted 1:300) for 30 minutes, followed by the incubation with Streptavidin-HRP (Millipore) for 15 minutes. To detect aldosterone synthase, after antigen retrieval, slides were incubated with anti-CYP11B2 (1:1000) over-night at $4{ }^{\circ} \mathrm{C}$. After rinsing in PBS, the EnVision reagent (Dako, Carpinteria, CA) coupled with peroxidase-labeled polymer was incubated as secondary antibody for $30 \mathrm{~min}$. The proteins were visualized with 3,3'diaminobenzidine tetrahydrochloride and counterstained with hematoxylin. Immunoreactivity for CYP11B2 was assessed semiquantitatively by the McCarthy H-score, with all tumors examined under a $20 \times$ objective. In each field the percentage of immunopositive cells was assessed and then multiplied by a factor (1-3) according to the intensity of the immunopositivity ( 1 = weak; 2 = intermediate; 3 = strong) (McCarty et al., 1985).

Immunofluorescence was performed as previously described (Gomez-Sanchez et al., 2014).

\subsection{Statistical analysis}

IBM SPSS statistics 21 was used for the statistical analyses. All data are presented as mean \pm SD or 25 th to 75 th percentile. Data were analyzed by the Kolmogorov-Smirnov and Shapiro-Wilk tests to determine their distribution. Statistically significance between groups was calculated in normally distributed data by Student's $t$ test for independent samples or ANOVA, in non-normally distributed data by Kruskal-Wallis and Mann-Whitney U test. The chisquare test or the Fisher exact test was applied for qualitative variables. Correlations were evaluated by Pearson correlation analysis and Spearman's rank-order tests. A probability value of $\mathrm{P} \leq 0.05$ was considered statistically significant.

\section{Results}

\subsection{Macroscopic evaluation}

We assessed macroscopically 71 adrenals removed from patients affected by unilateral PA. On the basis of adrenal CT scanning, 56 patients showed a single nodule, 4 multiple nodules and in 11 patients adrenal CT scanning could not identify any adrenal mass. Of the 11 patients in whom adrenal CT scanning did not show any nodule, the final diagnosis at histopathological examination was single nodule in 4 patients and multinodular hyperplasia in 7 patients. Notably, 2 of the 11 patients without nodules on imaging had a CACNA1D mutation in agreement with previous reports that patients with CACNA1D mutations had smaller adenomas than those with KCNJ5 mutations or no mutations (Fernandes-Rosa et al., 2014; Azizan et al., 2013). Overall, the final histopathological diagnosis was single nodule in 55 adrenals and multinodular hyperplasia in 16 adrenals. Interestingly, the final diagnosis of single nodule was more frequent in female than in male patients $(\mathrm{F}=32 ; \mathrm{M}=23)$, while the multinodular hyperplasia appeared more common in males $(F=7 ; M=9)$. Despite the different sex distribution, the prevalence of KCNJ5 mutations did not differ between the two groups: single nodule $21 / 55$, multinodular hyperplasia 6/16.

\subsection{Microscopic evaluation}

Analysis of histological features according to mutational status was performed in all 71 adrenal gland samples. Overall, 61\% (43/ 71) APAs removed consisted mainly ( $\geq 50 \%$ ) of ZF-like cells (Fig. 1 , panel A), while 39\% (28/71) were mainly ZG-like cells (Fig. 1, panel B). Analysis of cellular composition according to the mutational status revealed that KCNJ5-mutated APAs were composed mainly of ZFlike cells compared with both wild-type and CACNA1D mutated tumors (both $\mathrm{P}<0.001$ ). All the three APAs carrying mutations in CACNA1D were composed mainly of ZG-like cells. Of note, in our series of APAs, we could not identify any relationship between the cellular composition of the tumors and the responsiveness of aldosterone to upright posture.

Clinical and biochemical correlates according to cellular composition are summarized in Table 1 . We observed that adrenal nodules composed mainly of ZF-like cells display higher prevalence of KCNJ5 mutation, higher prevalence of CYP11B1 expression and lower CYP11B2 expression than ZG-like nodules (as will be further detailed later).

In addition, we examined the non tumor adrenal cortex from the adrenal glands included in the study: 31 of the 69 available cases (45\%) contained extra-APA B2-CNs (Supplementary Fig. S1, panels A and B). Patients' characteristics, histopathology and immunohistochemistry of adrenals with or without extra-APA B2-CNs are shown in Table 2. Interestingly, patients without extra-APA B2-CNs displayed more frequently single nodules at adrenal CT scanning compared to patients with extra-APA B2-CNs (Table 2). Moreover, among AVS parameters, the suppression of the contralateral adrenal was significantly more frequent $(\mathrm{p}=0.039)$ and the LI was higher $(\mathrm{p}=0.018)$ in the subgroup without extra-APA B2-CNs compared to the subgroup with extra-APA B2-CNs.

\subsection{Immunohistochemical and immunofluorescence analysis}

The final steps in mineralocorticoid and glucocorticoid biosynthesis are catalyzed by two closely related mitochondrial enzymes: $11 \beta$-hydroxylase (encoded by CYP11B1 gene) and aldosterone synthase (encoded by CYP11B2 gene) (Gomez-Sanchez et al., 2014). Seventy-one adrenal glands removed for unilateral PA were immunohistochemically examined for $11 \beta$-hydroxylase and aldosterone synthase expression.

Sixty-nine major adrenal nodules were identified by macroscopic examination: immunolocalization of steroidogenic enzymes revealed that $61 / 69(88 \%)$ of the nodules showed a variable degree of CYP11B2 expression (median H-score 60, 25th-75th percentile 15-160). Adrenal nodules showing high expression of CYP11B2 (arbitrarily defined as $\mathrm{H}$-score $>160$, representing the 75 th percentile) were smaller, composed mainly of ZG-like cells (Fig. 1, panels $C$ and D) and with a higher prevalence of ATP1A1/ATP2B3 and CACNA1D mutations compared to adrenal nodules with a lower score 

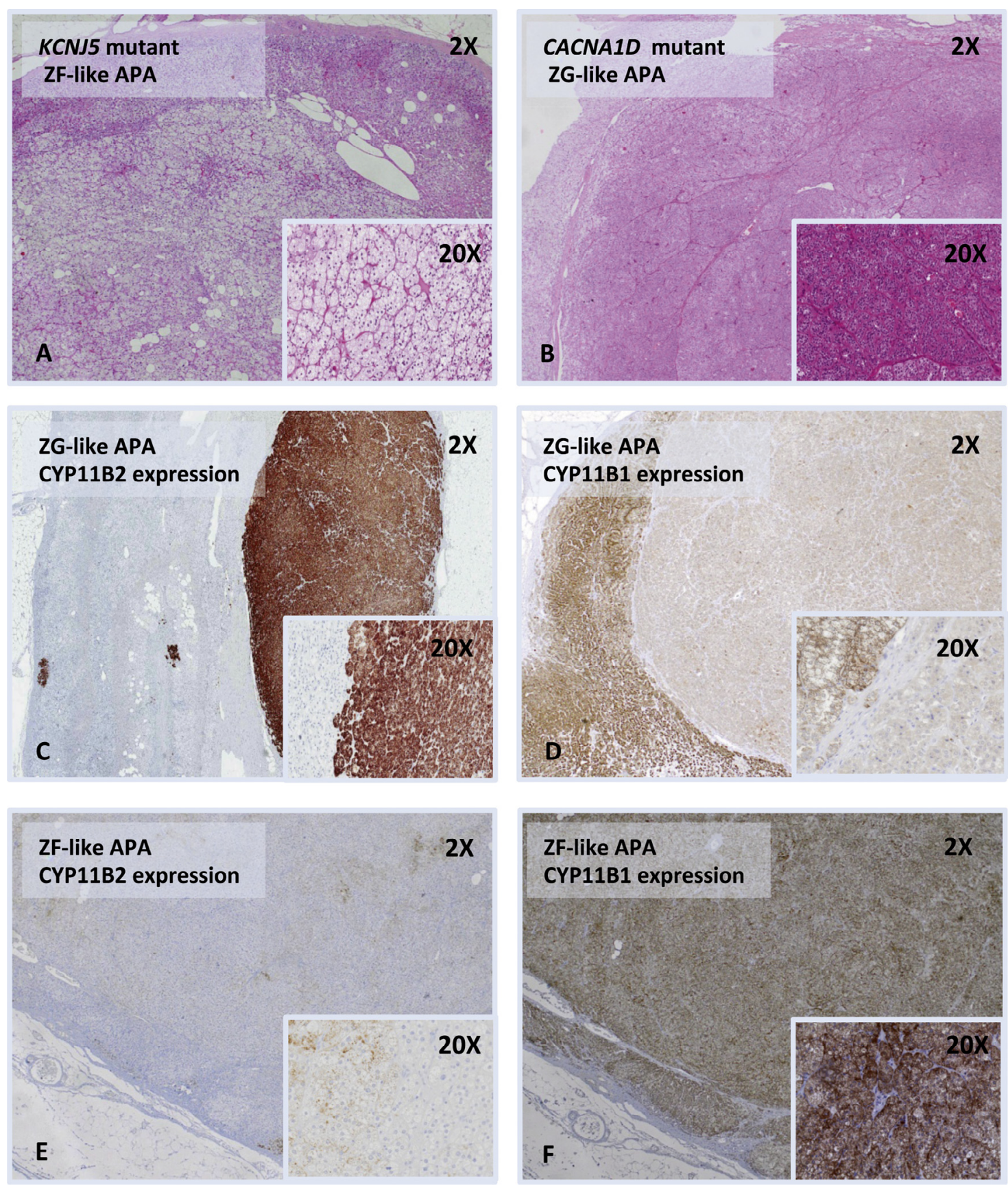

Fig. 1. Examples of histopathological findings in adrenal glands of patients with unilateral PA. Panel A: Hematoxylin-eosin staining of a KCNJ5-mutated APA with prevalent ZF-like cells; panel B: Hematoxylin-eosin staining of a CACNA1D-mutated APA composed mainly ZG-like cells; panel C: CYP11B2 immunostaining of an APA with prevalent ZG-like cells; panel D: CYP11B2 immunostaining of an APA with prevalent ZG-like cells; panel E: CYP11B2 immunostaining of an APA with prevalent ZF-like cells; panel F: CYP11B1 immunostaining of an APA with prevalent ZF-like cells.

(H-score $<160$, Table 3). Moreover, we observed a significant inverse correlation between CYP11B2 expression (H-score) and nodule size $(-0.308$, $\mathrm{p}$-value $=0.009)$. In addition, if CYP11B2 expression was corrected for tumor volume, we observed a significant positive correlation with plasma aldosterone and ARR. Two adrenals with a final diagnosis of multinodular hyperplasia showed negative immunostaining for CYP11B2 in the main nodule, but the enzyme was expressed in a different smaller nodule (Supplementary Fig. S1, panels $C$ and D). Of the eight samples not expressing CYP11B2 (Hscore $=0$ ), one case underwent adrenalectomy without AVS for suspicion of aldosterone-producing adrenocortical carcinoma (hyperaldosteronism and hypertension were cured by adrenalectomy).
Overall, of the 8 patients showing negative CYP11B2 immunostaining, 7 expressed CYP11B1 in the main nodule, 2 had extra-APA B2-CNs in the adjacent adrenal cortex, 6 were composed mainly of ZF-like cells, 4 had a final diagnosis of adenoma, 4 of multinodular hyperplasia. Genotyping revealed that 5/8 were wild-type and 3/8 had a mutation in KCNJ5. Overall, clinical and biochemical parameters of patients expressing or not aldosterone synthase did not differ significantly from each other (as detailed in Supplementary Tables S2 and S3).

H-score of CYP11B2 expression was significantly lower in adrenal nodules expressing CYP11B1 compared to APAs not expressing CYP11B1 (155 [45-262] vs 40 [10-120], p = 0.001) (Table 4) and the 
Table 1

Clinical and biochemical parameters of patients included in the study, immunohistochemistry and genotyping according to the cellular composition.

\begin{tabular}{|c|c|c|c|}
\hline \multirow[t]{2}{*}{ Parameter } & \multicolumn{2}{|c|}{ Cellular composition } & \multirow[t]{2}{*}{ P value } \\
\hline & $\begin{array}{l}\text { ZF-like } \\
(\mathrm{n}=43)\end{array}$ & $\begin{array}{l}\text { ZG-like } \\
(\mathrm{n}=28)\end{array}$ & \\
\hline Age (years) & $46 \pm 11$ & $49 \pm 12$ & 0.4 \\
\hline $\operatorname{Sex}(M / F)$ & $18 / 25$ & $14 / 14$ & 0.6 \\
\hline $\mathrm{SBP}(\mathrm{mmHg})$ & $170 \pm 26$ & $175 \pm 27$ & 0.5 \\
\hline $\mathrm{DBP}(\mathrm{mmHg})$ & $105 \pm 12$ & $105 \pm 14$ & 0.9 \\
\hline Plasma K+ $(\mathrm{mEq} / \mathrm{L})$ & $2.9 \pm 0.8$ & $2.9 \pm 0.6$ & 0.7 \\
\hline $\mathrm{PRA}(\mathrm{ng} / \mathrm{mL} / \mathrm{h})$ & $0.2[0.2-0.3]$ & $0.2[0.2-0.3]$ & 0.3 \\
\hline Plasma aldosterone (ng/dL) & $45.1[33.7-60.0]$ & $49.3[38.2-67.2]$ & 0.4 \\
\hline Adenoma size $(\mathrm{mm})$ & $1.8 \pm 0.8$ & $1.5 \pm 1.1$ & 0.2 \\
\hline Adrenal CT scanning & & & 0.2 \\
\hline Single nodule, $\mathrm{n}(\%)$ & $34(79 \%)$ & $22(79 \%)$ & \\
\hline Multiple nodules, $\mathrm{n}(\%)$ & $4(9 \%)$ & $0(0 \%)$ & \\
\hline No nodules, $\mathrm{n}(\%)$ & $5(12 \%)$ & $6(21 \%)$ & \\
\hline Lateralization index & $9.3[5.0-12.9]$ & $9.3[4.5-19.4]$ & 0.8 \\
\hline Contralateral suppression (yes/no) & $32 / 9$ & $21 / 5$ & 1.0 \\
\hline \multicolumn{4}{|l|}{ Final diagnosis } \\
\hline Single nodule, n (\%) & $33(77 \%)$ & $22(79 \%)$ & 0.5 \\
\hline Multinodular hyperplasia, n (\%) & $10(23 \%)$ & $6(21 \%)$ & \\
\hline H-score B2 & $40[10-90]$ & $170[43-263]$ & $<0.001$ \\
\hline Corrected H-score B2 & $52[10-275]$ & $133[46-375]$ & 0.3 \\
\hline extra-APA B2-CNs (yes/no) & $19 / 22$ & $12 / 16$ & 0.8 \\
\hline CYP11B1 expression (yes/no) & $37 / 6$ & $10 / 18$ & $<0.001$ \\
\hline Postural test (positive/negative) & $9 / 23$ & $7 / 12$ & 0.4 \\
\hline \multicolumn{4}{|l|}{ Mutational status } \\
\hline WT, n (\%) & $16(37 \%)$ & $19(68 \%)$ & $<0.001^{\mathrm{a}}$ \\
\hline KCNJ5 mut, n (\%) & $25(58 \%)$ & $2(7 \%)$ & $0.19^{\mathrm{b}}$ \\
\hline ATP1A1-ATP2B3-CACNA1D mut, n (\%) & $2(5 \%)$ & $7(25 \%)$ & \\
\hline
\end{tabular}

SBP, systolic blood pressure; DBP, diastolic blood pressure; PRA, plasma renin activity; extra-APA B2-CNs, extra-APA CYP11B2-positive cell nests; ZF-like, zona fasciculata like; ZG-like, zona glomerulosa like.

a KCNJ5 vs WT and KCNJ5 vs ATP1A1-ATP2B3-CACNA1D.

b WT vs ATP1A1-ATP2B3-CACNA1D.

Table 2

Clinical and biochemical parameters, immunohistochemistry, genotyping and cellular composition according to the presence or absence of extra-APA CYP11B2-positive cell nests.

\begin{tabular}{|c|c|c|c|}
\hline \multirow[t]{2}{*}{ Parameter } & \multicolumn{2}{|c|}{ Extra-APA CYP11B2-positive cell nest } & \multirow[t]{2}{*}{ P value } \\
\hline & $\begin{array}{l}\text { Present } \\
(\mathrm{n}=31)\end{array}$ & $\begin{array}{l}\text { Absent } \\
(\mathrm{n}=38)\end{array}$ & \\
\hline Age (years) & $48 \pm 9$ & $46 \pm 13$ & 0.4 \\
\hline $\operatorname{Sex}(M / F)$ & $11 / 20$ & $20 / 18$ & 0.2 \\
\hline $\mathrm{SBP}(\mathrm{mmHg})$ & $174 \pm 24$ & $171 \pm 27$ & 0.6 \\
\hline $\mathrm{DBP}(\mathrm{mmHg})$ & $106 \pm 12$ & $104 \pm 14$ & 0.5 \\
\hline Plasma $\mathrm{K}^{+}(\mathrm{mEq} / \mathrm{L})$ & $2.9 \pm 0.7$ & $2.9 \pm 0.7$ & 0.9 \\
\hline PRA (ng/mL/h) & $0.25[0.20-0.50]$ & $0.20[0.20-0.30]$ & 0.5 \\
\hline Plasma aldosterone (ng/dL) & $44.8[29-57.9]$ & $51.9[34.6-65.6]$ & 0.2 \\
\hline Adenoma size $(\mathrm{mm})$ & $1.59 \pm 0.7$ & $1.68 \pm 1.0$ & 0.7 \\
\hline \multicolumn{4}{|l|}{ Adrenal CT scanning } \\
\hline Single nodule, $\mathrm{n}(\%)$ & $20(64 \%)$ & $35(92 \%)$ & \multirow[t]{3}{*}{0.013} \\
\hline Multiple nodules, n (\%) & $3(10 \%)$ & $0(0 \%)$ & \\
\hline No nodules, $\mathrm{n}(\%)$ & $8(26 \%)$ & $3(8 \%)$ & \\
\hline Lateralization index & $5.5[4.4-11.8]$ & $11.0[5.6-21.3]$ & 0.018 \\
\hline Contralateral suppression (yes/no) & $20 / 10$ & $31 / 4$ & 0.039 \\
\hline Final diagnosis & & & 0.5 \\
\hline Single nodule, n (\%) & $23(74 \%)$ & $31(82 \%)$ & \\
\hline Multinodular hyperplasia, n (\%) & $8(26 \%)$ & $7(18 \%)$ & \\
\hline H-score CYP11B2 & $80[20-140]$ & $60[10-180]$ & 0.7 \\
\hline Corrected H-score CYP11B2 & $86[43-424]$ & $110[10-348]$ & 0.7 \\
\hline Cellular composition (ZF-like/ZG-like) & $19 / 12$ & $22 / 16$ & 0.810 \\
\hline CYP11B1 expression (yes/no) & $20 / 11$ & $25 / 13$ & 0.556 \\
\hline Postural test (positive/negative) & $8 / 14$ & $8 / 19$ & 0.761 \\
\hline \multicolumn{4}{|l|}{ Mutational status } \\
\hline WT, n (\%) & $13(42 \%)$ & $20(53 \%)$ & \multirow[t]{3}{*}{0.627} \\
\hline KCNJ5 mut, n (\%) & $13(42 \%)$ & $14(37 \%)$ & \\
\hline ATP1A1-ATP2B3-CACNA1D mut, n (\%) & $5(16 \%)$ & $4(10 \%)$ & \\
\hline
\end{tabular}

SBP, systolic blood pressure; DBP, diastolic blood pressure; PRA, plasma renin activity; ZF-like, zona fasciculata like; ZG-like, zona glomerulosa like. 
Table 3

Clinical and biochemical parameters, immunohistochemistry, genotyping and cellular composition according to the level (H-score) of CYP11B2 expression in the adrenal nodules.

\begin{tabular}{|c|c|c|c|}
\hline \multirow[t]{2}{*}{ Parameter } & \multicolumn{2}{|c|}{ CYP11B2 expression in adrenal nodules } & \multirow[t]{2}{*}{ P value } \\
\hline & $\begin{array}{l}\text { H-score } 0-160 \\
(\mathrm{n}=55)\end{array}$ & $\begin{array}{l}\text { H-score } 161-300 \\
(n=16)\end{array}$ & \\
\hline Age (years) & $48 \pm 12$ & $44 \pm 12$ & 0.4 \\
\hline $\operatorname{Sex}(M / F)$ & $21 / 34$ & $11 / 5$ & 0.045 \\
\hline $\mathrm{SBP}(\mathrm{mmHg})$ & $171 \pm 26$ & $176 \pm 24$ & 0.5 \\
\hline $\mathrm{DBP}(\mathrm{mmHg})$ & $104 \pm 13$ & $109 \pm 13$ & 0.2 \\
\hline Plasma $\mathrm{K}^{+}(\mathrm{mEq} / \mathrm{L})$ & $2.9 \pm 0.8$ & $2.9 \pm 0.5$ & 0.9 \\
\hline PRA (ng/mL/h) & $0.2[0.2-0.3]$ & $0.25[0.2-0.52]$ & 0.1 \\
\hline Plasma aldosterone (ng/dL) & $45.3[33.7-58.9]$ & $51.4[38.8-68.9]$ & 0.2 \\
\hline Adenoma size $(\mathrm{mm})$ & $1.55[1.0-2.42]$ & $1.0[1.0-1.47]$ & 0.011 \\
\hline \multicolumn{4}{|l|}{ Adrenal CT scanning } \\
\hline Single nodule, n (\%) & $43(78 \%)$ & $13(81 \%)$ & 0.5 \\
\hline Multiple nodules, n (\%) & $4(7 \%)$ & $0(0 \%)$ & \\
\hline No nodules, $\mathrm{n}(\%)$ & $8(15 \%)$ & $3(19 \%)$ & \\
\hline Lateralization index & $9.4[5.0-17.0]$ & $7.2[4.3-14.6]$ & 0.4 \\
\hline Contralateral suppression (yes/no) & $41 / 10$ & $12 / 4$ & 0.7 \\
\hline \multicolumn{4}{|l|}{ Final diagnosis } \\
\hline Single nodule, $\mathrm{n}(\%)$ & $41(74.5 \%)$ & $14(87.5 \%)$ & 0.1 \\
\hline Multinodular hyperplasia, n (\%) & $14(25.5 \%)$ & $2(12.5 \%)$ & \\
\hline CYP11B1 expression (yes/no) & $42 / 13$ & $5 / 11$ & 0.002 \\
\hline Cellular composition (ZF-like/ZG-like) & $41 / 14$ & $2 / 14$ & $<0.001$ \\
\hline Extra-APA B2-CN (yes/no) & $26 / 27$ & $5 / 11$ & 0.2 \\
\hline Postural test (positive/negative) & $13 / 27$ & $3 / 8$ & 1.0 \\
\hline \multicolumn{4}{|l|}{ Mutational status } \\
\hline WT, n (\%) & $24(44 \%)$ & $11(69 \%)$ & $0.001^{\mathrm{a}}$ \\
\hline KCNJ5 mut, n (\%) & $26(47 \%)$ & $1(6 \%)$ & $0.009^{b}$ \\
\hline ATP1A1-ATP2B3-CACNA1D mut, n (\%) & $5(9 \%)$ & $4(25 \%)$ & $0.46^{c}$ \\
\hline
\end{tabular}

SBP, systolic blood pressure; DBP, diastolic blood pressure; PRA, plasma renin activity; Extra-APA B2-CN, CYP11B2positive cell nests; ZF-like, zona fasciculate like; ZG-like, zona glomerulosa like.

a KCNJ5 mut vs WT.

b 0.009 KCNJ5 mut vs ATP1A1-ATP2B3-CACNA1D mut.

c WT vs ATP1A1-ATP2B3-CACNA1D mut.

expression of the two hydroxylases were inversely correlated (correlation coefficient $-0.395, \mathrm{p}=0.001)$. Overall, $47 / 69(68 \%)$ of the adrenal nodules identified by macroscopic examination displayed positive immunostaining for CYP11B1. Forty-one out of 47 adrenal nodules expressing CYP11B1 were larger than $1 \mathrm{~cm}$ and subclinical cortisol hypercortisolism (defined as failure to suppress plasma cortisol to less than $50 \mathrm{nmol} / \mathrm{L}$ after $1 \mathrm{mg}$-overnight dexamethasone) was excluded. APA patients expressing CYP11B1 were more frequently females ( $\mathrm{p}=0.040$ ), carrying mutations in KCNJ5 and composed of ZF-like cells compared with APA patients not expressing CYP11B1 (Fig. 1, panels E and F), as detailed in Table 4. Among clinical and biochemical parameters, APA patients not expressing CYP11B1 had higher SBP and lower potassium compared to patients expressing CYP11B1 in the adrenal nodule, despite neither absolute aldosterone levels nor ARR differing between the two groups (Table 4).

We also performed immunofluorescence staining in some of our APA (Supplementary Fig. S2): most cells expressed either CYP11B2 (panel A) or CYP11B1 (panel B) and only a few cells co-expressed both enzymes (panel C).

\section{Discussion}

Over the last few years great strides have been made in our understanding of the genetics and pathophysiology of sporadic and familial PA. The activation of the calcium signaling pathway is critical for aldosterone secretion and represents the convergence point of the different electrical and ionic alterations induced by the recently reported somatic mutations in APAs (Azizan et al., 2013; Beuschlein et al., 2013; Boulkroun et al., 2012; Choi et al., 2011; Monticone et al., 2014a).
The final step in mineralocorticoid biosynthesis is catalyzed by aldosterone synthase (encoded by CYP11B2), a mitochondrial enzyme which shares 95\% amino acid sequence identity with $11 \beta$ hydroxylase (encoded by CYP11B1), that converts 11-deoxycortisol to cortisol (Mulatero et al., 1998). Taking advantage of the recently developed monoclonal antibodies (Gomez-Sanchez et al., 2014), in the present study we investigated the relationship between the presence of somatic mutations and the different cell histology and immunohistochemical staining profiles for $11 \beta$-hydroxylase and aldosterone synthase in a large cohort of 71 adrenal glands removed for unilateral PA.

APA and BAH, the two most common forms of primary aldosteronism, were originally considered distinct conditions. However, over the last few years, this distinction began to blur and recent evidence point toward a pathological continuum between APA and BAH (Boulkroun et al., 2010; Omura et al., 2002). In fact, in the present study, we observed that $23 \%$ of the assessed adrenals, preoperatively diagnosed as unilateral PA, showed multiple nodules and a final diagnosis of multinodular hyperplasia was made.

In agreement with previous reports (Azizan et al., 2012, 2013), our results suggest that KCNJ5 mutations can at least partially differentiate a subpopulation of female APAs, which are composed of large vacuolated cells and express CYP11B1 as a biomarker of the zona fasciculata. In our cohort, the mean size of the adenomas did not differ significantly according to the mutational status. However, we observed a tendency toward smaller dimensions in CACNA1Dmutant tumors compared to wild-type or KCNJ5-mutants, not statistically significant due to the small sample size, highlighting the need for AVS in subtype differentiation, in particular in those patients with negative adrenal CT findings (Monticone et al., 2014c). As outlined by Azizan et al. (2013), adenomas harboring ATP1A1, 
Table 4

Clinical and biochemical parameters, immunohistochemistry, genotyping and cellular composition according to the presence or absence of CYP11B1 expression in the adrenal nodules.

\begin{tabular}{|c|c|c|c|}
\hline \multirow[t]{2}{*}{ Parameter } & \multicolumn{2}{|c|}{ CYP11B1 expression in adrenal nodules } & \multirow[t]{2}{*}{ P value } \\
\hline & $\begin{array}{l}\text { Expressed } \\
(\mathrm{n}=47)\end{array}$ & $\begin{array}{l}\text { Not expressed } \\
(\mathrm{n}=24)\end{array}$ & \\
\hline Age (years) & $48 \pm 10$ & $44 \pm 13$ & 0.2 \\
\hline $\operatorname{Sex}(M / F)$ & $17 / 30$ & $15 / 9$ & 0.045 \\
\hline $\mathrm{SBP}(\mathrm{mmHg})$ & $167 \pm 22$ & $182 \pm 29$ & 0.018 \\
\hline $\mathrm{DBP}(\mathrm{mmHg})$ & $103 \pm 12$ & $108 \pm 14$ & 0.1 \\
\hline Plasma K+ $\left.\mathrm{K}^{+} \mathrm{mEq} / \mathrm{L}\right)$ & $3.0 \pm 0.6$ & $2.6 \pm 0.8$ & 0.013 \\
\hline PRA (ng/mL/h) & $0.2[0.2-0.3]$ & $0.2[0.2-0.3]$ & 0.3 \\
\hline Plasma aldosterone (ng/dL) & $45.5[33.9-58.0]$ & $50.5[31.3-72.8]$ & 0.4 \\
\hline ARR & 226 [115-359] & $190[104-415]$ & 0.8 \\
\hline Adenoma size (mm) & $1.8 \pm 1.0$ & $1.4 \pm 0.6$ & 0.06 \\
\hline Adrenal CT scanning & & & 0.9 \\
\hline Single nodule, $\mathrm{n}(\%)$ & $34(79 \%)$ & $19(79 \%)$ & \\
\hline Multiple nodules, n (\%) & $3(6 \%)$ & $1(4 \%)$ & \\
\hline No nodules, $\mathrm{n}(\%)$ & $7(15 \%)$ & $4(17 \%)$ & \\
\hline Lateralization index & $7.6[4.8-12.2]$ & $14.2[4.8-27.2]$ & 0.059 \\
\hline Contralateral suppression (yes/no) & $35 / 11$ & $18 / 3$ & 0.5 \\
\hline Final diagnosis & & & 0.5 \\
\hline Single nodule, $\mathrm{n}(\%)$ & $35(74.5 \%)$ & $20(83 \%)$ & \\
\hline Multinodular hyperplasia, n (\%) & $12(25.5 \%)$ & $4(17 \%)$ & \\
\hline H-score CYP11B2 & $40[10-120]$ & $155[45-262]$ & 0.001 \\
\hline Corrected H-score CYP11B2 & $52[10-274]$ & $141[51-375]$ & 0.2 \\
\hline Cellular composition (ZF-like/ZG-like) & $37 / 10$ & $6 / 18$ & $<0.001$ \\
\hline Extra-APA B2-CN (yes/no) & $20 / 25$ & $11 / 13$ & 1.0 \\
\hline Postural test (positive/negative) & $11 / 25$ & $5 / 10$ & 0.7 \\
\hline \multicolumn{4}{|l|}{ Mutational status } \\
\hline WT, n (\%) & $21(45 \%)$ & $14(58 \%)$ & $0.047^{\mathrm{a}}$ \\
\hline KCNJ5 mut, n (\%) & $23(49 \%)$ & $4(17 \%)$ & $0.0062^{\mathrm{b}}$ \\
\hline ATP1A1-ATP2B3-CACNA1D mut, n (\%) & $3(6 \%)$ & $6(25 \%)$ & $0.14^{\mathrm{c}}$ \\
\hline
\end{tabular}

SBP, systolic blood pressure; DBP, diastolic blood pressure; PRA, plasma renin activity; extra-APA B2-CN, CYP11B2positive cell nests; ZF-like, zona fasciculata like; ZG-like, zona glomerulosa like.

a KCNJ5 mut vs WT.

b KCNJ5 mut vs ATP1A1-ATP2B3-CACNA1D mut.

c WT vs ATP1A1-ATP2B3-CACNA1D mut.

ATP2B3 and CACNA1D mutations arise more frequently from zona glomerulosa cells and display stronger positive immunostaining for CYP11B2 compared to KCNJ5 mutant APAs. The so-called wildtype tumors are probably a heterogeneous group of adenomas carrying mutations in other so far unidentified genes. It is conceivable that mutations of genes regulating ion exchange and in particular calcium entry across the plasma membrane of cells of the zona glomerulosa, together with genes involved in the regulation of cell growth and apoptosis, may be responsible for the APA formation and regulation of aldosterone production in this group of APA. We did not observe differences among clinical and biochemical parameters between wild-type and mutated APAs (Williams et al., 2014).

The prevalence of CT-undetectable APAs, which can be diagnosed only by AVS, is reported to be between $13 \%$ and $30 \%$ in different series (Young et al., 2004). Ono et al. explored the status of aldosterone production in small APAs, demonstrating that the immunoreactivity of CYP11B2 was higher in microadenomas compared to macroadenomas and the H-score of CYP11B2 inversely correlated with the tumor area (Ono et al., 2014). Similarly, in our cohort, CYP11B2 H-score displayed a negative correlation with adenoma size and CYP11B1 expression and, after correction for tumor volume, a positive correlation with plasma aldosterone levels and ARR, which reflect the biochemical severity of the disease as reported before (Nanba et al., 2013).

Interestingly, two samples showed negative CYP11B2 immunostaining in the main nodule and strong positive immunostaining in a secondary small nodule, indicating that the big nodulation detected by adrenal CT scanning (despite clear lateralization at AVS) may not always be the source of aldosterone overproduction in PA patients. This consideration, along with the reported recurrence of PA after adrenal-sparing surgery (Ishidoya et al., 2005), suggests that laparoscopic total adrenalectomy is preferable over adenectomy.

It may be surprising the observation that some APAs did not show detectable CYP11B2 immunostaining in the main or secondary nodules; this has been found previously by other scientists: Dekkers et al. (2014) found 9.6\% APA that were CYP11B2-negative and no other nodules expressing aldosterone-synthase; furthermore, in a previous study Lenzini et al. (2007) reported 37.6\% of APA with a CYP11B2 gene expression inferior to normal adrenals. We performed a real-time rt-PCR for CYP11B2 and in all APAs with negative staining we could nonetheless demonstrate a detectable expression of CYP11B2. It is conceivable that in some cases CYP11B2 expression is not high enough to be detectable with immunohistochemistry; however, a high number of cells producing small quantity of aldosterone could result in a final increased aldosterone production. After adrenalectomy, all these patients displayed normalization of PRA, aldosterone, ARR and potassium levels, and normalization or marked reduction of blood pressure levels.

We observed that multinodular hyperplasia is a frequent cause of unilateral PA. This finding is in agreement with a recent study by lacobone et al. (2012) reporting a even higher prevalence of $65 \%$. It is conceivable that those groups that do not systematically use AVS to discriminate between unilateral and bilateral PA may adrenalectomize only (or prevalently) patients with a single unilateral nodule at CT scan, therefore having a relatively reduced probability of multinodular hyperplasia at pathological examination. We did 
not observe differences in the final biochemical or clinical outcome between patients having multinodular hyperplasia or APA as shown by others (Iacobone et al., 2012). No evidence of recurrent PA has been observed in the follow-up.

Extra-APA B2-CNs have been identified both in normal adrenals and in the context of the adjacent cortex to an APA as morphologically and immunohistochemically distinct structures expressing CYP11B2, but not CYP11B1. Despite their functional significance remaining unclear, some authors have hypothesized that they might give rise to an APA (Nishimoto et al., 2010). Overall, we observed extra-APA B2-CNs in $45 \%$ of the removed adrenal glands. Adrenals bearing extra-APA B2-CNs display more frequently multiple nodules at adrenal CT scanning, lower LI and lower prevalence of contralateral adrenal suppression compared to adrenal glands in which extra-APA B2-CNs could not be identified. Despite the clinical significance of extra-APA B2-CNs remaining poorly elucidated, it is conceivable that in these cases PA was originally a bilateral disease and subsequently, in the context of a multinodular adrenal gland, an extra-APA B2-CN has given rise to an APA thus becoming dominant (Gomez-Sanchez and Gomez-Sanchez, 2012).

Two subtypes of patients with APA have been identified, based on different aldosterone response to up-right posture (AII-responsive and AII-unresponsive APAs). The different cellular composition (predominance of ZF-like cells or ZG-like cells) has been suggested by some authors to be responsible for this distinct biochemical behavior (Tunny et al., 1991), but was not confirmed in other series (Fallo et al., 1998; Mune et al., 1993). In our cohort, there were no significant differences between these two groups with respect to the cellular composition, mutational status, clinical and biochemical characteristics, CYP11B1 and CYP11B2 immunostaining.

\section{Conclusions}

In conclusion, our findings indicate that combination of genotyping and immunohistochemistry improves the final histopathological diagnosis between single nodule and multinodular hyperplasia of the assessed adrenals. Most cases preoperatively classified as an APA are morphologically heterogeneous and the identification of somatic mutations in KCNJ5, ATP1A1, ATP2B3 and CACNA1D associated with the use of the recently developed monoclonal antibodies for aldosterone synthase and 11ß-hydroxylase at least partially accounts for this histopathological heterogeneity.

\section{Acknowledgements}

P.M. and I.C. received a grant from the Italian Ministry of Instruction, University and Research (grant ex-60\%, 2013); C.E.G.-S. is supported by NIH grant R01HL27255.

We thank Dr. Giuseppe Giraudo, MD and Prof. Mauro Maccario, MD for help in diagnosis and management of our PA patients; we thank Dr. Domenica Schiavone, PhD for help in the DNA extraction, sequencing and hormone assays.

\section{Appendix: Supplementary material}

Supplementary data to this article can be found online at doi:10.1016/j.mce.2015.04.022.

\section{References}

Azizan, E.A., Lam, B.Y., Newhouse, S.J., Zhou, J., Kuc, R.E., Clarke, J., et al., 2012. Microarray, qPCR, and KCNJ5 sequencing of aldosterone-producing adenomas reveal differences in genotype and phenotype between zona glomerulosa- and zona fasciculata-like tumors. J. Clin. Endocrinol. Metab. 97, E819-E829.

Azizan, E.A., Poulsen, H., Tuluc, P., Zhou, J., Clausen, M.V., Lieb, A., et al., 2013. Somatic mutations in ATP1A1 and CACNA1D underlie a common subtype of adrenal hypertension. Nat. Genet. 45, 1055-1060.
Beuschlein, F., Boulkroun, S., Osswald, A., Wieland, T., Nielsen, H.N., Lichtenauer, U.D. et al. 2013. Somatic mutations in ATP1A1 and ATP2B3 lead to aldosteroneproducing adenomas and secondary hypertension. Nat. Genet. 45, 440-444.

Boulkroun, S., Samson-Couterie, B., Dzib, J.F., Lefebvre, H., Louiset, E., Amar, L., et al., 2010. Adrenal cortex remodeling and functional zona glomerulosa hyperplasia in primary aldosteronism. Hypertension 56, 885-892.

Boulkroun, S., Beuschlein, F., Rossi, G.P., Golib-Dzib, J.F., Fischer, E., Amar, L., et al., 2012. Prevalence, clinical, and molecular correlates of KCNJ5 mutations in primary aldosteronism. Hypertension 59, 592-598.

Choi, M., Scholl, U.I., Yue, P., Björklund, P., Zhao, B., Nelson-Williams, C., et al., 2011. $\mathrm{K}+$ channel mutations in adrenal aldosterone-producing adenomas and hereditary hypertension. Science 331, 768-772.

Dekkers, T., Ter Meer, M., Lenders, J.W.M., Hermus, A.R.M., Schultze Kool, L., Langenhuijsen, J.F., et al., 2014. Adrenal nodularity and somatic mutations in primary aldosteronism: one node is the culprit? J. Clin. Endocrinol. Metab. 99, E1341-E1351.

Fallo, F., Barzon, L., Biasi, F., Altavilla, G., Boscaro, M., Sonino, N., 1998. Zona fasciculata-like histotype and aldosterone response to upright posture are not related in aldosterone-producing adenoma. Exp. Clin. Endocrinol. Diabetes 106, $74-78$.

Fallo, F., Bertello, C., Tizzani, D., Fassina, A., Boulkroun, S., Sonino, N., et al., 2011. Concurrent primary aldosteronism and subclinical cortisol hypersecretion: a prospective study. J. Hypertens. 29, 1773-1777.

Fernandes-Rosa, F.L., Williams, T.A., Riester, A., Steichen, O., Beuschlein, F., Boulkroun, S., 2014. Genetic spectrum and clinical correlates of somatic mutations in aldosterone-producing adenoma. Hypertension 64, 354-361.

Funder, J.W., Carey, R.M., Fardella, C., Gomez-Sanchez, C.E., Mantero, F., Stowasser, M., et al., 2008. Endocrine society. Case detection, diagnosis, and treatment of patients with primary aldosteronism: an endocrine society clinical practice guideline. J. Clin. Endocrinol. Metab. 93, 3266-3281.

Gomez-Sanchez, C.E., Gomez-Sanchez, E.P., 2012. Mutations of the potassium channel KCNJ5 causing aldosterone-producing adenomas: one or two hits? Hypertension 59, 196-197.

Gomez-Sanchez, C.E. Oi, X., Velarde-Miranda, C., Plonczynski, M.W., Parker, C.R., Rainey, W., et al., 2014. Development of monoclonal antibodies against human CYP11B1 and CYP11B2. Mol. Cell. Endocrinol. 383, 111-117.

Iacobone, M., Citton, M., Viel, G., Boetto, R., Bonadio, I., Tropea, S., et al., 2012. Unilateral adrenal hyperplasia: a novel cause of surgically correctable primary hyperaldosteronism. Surgery 152, 1248-1255.

Ishidoya, S., Ito, A., Sakai, K., Satoh, M., Chiba, Y., Sato, F., et al., 2005. Laparoscopic partial versus total adrenalectomy for aldosterone producing adenoma. J. Urol. 174, 40-43.

Lenzini, L., Seccia, T.M., Aldighieri, E., Belloni, A.S., Bernante, P., Giuliani, L., et al., 2007. Heterogeneity of aldosterone-producing adenomas revealed by a whole transcriptome analysis. Hypertension 50, 1106-1113.

McCarty, K.S., Jr., Miller, L.S., Cox, E.B., Konrath, J., McCarty, K.S., Sr., 1985. Estrogen receptor analyses. Correlation of biochemical and immunohistochemical methods using monoclonal antireceptor antibodies. Arch. Pathol. Lab. Med. 109, 716-721.

Monticone, S., Satoh, F., Giacchetti, G., Viola, A., Morimoto, R., Kudo, M., et al., 2012. Effect of adrenocorticotropic hormone stimulation during adrenal vein sampling in primary aldosteronism. Hypertension 59, 840-846.

Monticone, S., Else, T., Mulatero, P., Williams, T.A., Rainey, W.E., et al., 2014a. Understanding primary aldosteronism: impact of next generation sequencing and expression profiling. Mol. Cell. Endocrinol. 399C, 311-320.

Monticone, S., Satoh, F., Viola, A., Fischer, E., Vonend, O., Bernini, G., et al., 2014b. Aldosterone suppression on contralateral adrenal during adrenal vein sampling does not predict blood pressure response after adrenalectomy. J. Clin. Endocrinol. Metab. 99, 4158-4166.

Monticone, S., Viola, A., Rossato, D., Veglio, F., Reincke, M., Gomez-Sanchez, C., et al., 2014c. Adrenal vein sampling in primary aldosteronism: towards a standardised protocol. Lancet Diabetes Endocrinol. doi:10.1016/S2213-8587(14)70069-5; Epub ahead of print.

Mulatero, P., Curnow, K.M., Aupetit-Faisant, B., Foekling, M., Gomez-Sanchez, C., Veglio, F., et al., 1998. Recombinant CYP11B genes encode enzymes that can catalyze conversion of 11-deoxycortisol to cortisol, 18-hydroxycortisol, and 18-oxocortisol. J. Clin. Endocrinol. Metab. 83, 3996-4001.

Mulatero, P., Stowasser, M., Loh, K.C., Fardella, C.E., Gordon, R.D., Mosso, L., et al., 2004 Increased diagnosis of primary aldosteronism, including surgically correctable forms, in centers from five continents. J. Clin. Endocrinol. Metab. 89, 10451050.

Mulatero, P. Bertello, C., Rossato, D., Mengozzi, G., Milan, A., Garrone, C, et al, 2008. Roles of clinical criteria, computed tomography scan, and adrenal vein sampling in differential diagnosis of primary aldosteronism subtypes. J. Clin. Endocrinol. Metab. 93, 1366-1371.

Mulatero, P., Monticone, S., Bertello, C., Viola, A., Tizzani, D., Iannaccone, A., et al., 2013. Long-term cardio- and cerebrovascular events in patients with primary aldosteronism. J. Clin. Endocrinol. Metab. 98, 4826-4833.

Mune, T., Morita, H., Yasuda, K., Yamakita, N., Miura, K., 1993. Reduced response to metoclopramide and anomalous rising response to upright posture of plasma aldosterone concentration in Japanese patients with aldosterone-producing adenoma. J. Clin. Endocrinol. Metab. 77, 1020-1027.

Nakamura, Y., Felizola, S., Satoh, F., Konosu-Fukaya, S., Hironobu, S., 2014. Dissecting the molecular pathways of primary aldosteronism. Pathol. Int. 64, 482-489.

Nanba, K., Tsuiki, M., Sawai, K., Mukai, K., Nishimoto, K., Usui, T., et al., 2013. Histopathological diagnosis of primary aldosteronism using CYP11B2 immunohistochemistry. J. Clin. Endocrinol. Metab. 98, 1567-1574. 
Neville, A.M., O'Hare, M.J., 1985. Histopathology of the human adrenal cortex. Clin. Endocrinol. Metab. 14, 791-820.

Nishimoto, K., Nakagawa, K., Li, D., Kosaka, T., Oya, M., Mikami, S., et al., 2010. Adrenocortical zonation in humans under normal and pathological conditions. J. Clin. Endocrinol. Metab. 95, 2296-2305.

Omura, M., Sasano, H., Fujiwara, T., Yamaguchi, K., Nishikawa, T., 2002. Unique cases of unilateral hyperaldosteronemia due to multiple adrenocortical micronodules, which can only be detected by selective adrenal venous sampling. Metabolism 51, 350-355.

Ono, Y., Nakamura, Y., Maekawa, T., Felizola, S.J.A., Morimoto, R., Iwakura, Y., et al. 2014. Different expression of 11ß-hydroxylase and aldosterone synthase between aldosterone-producing microadenomas and macroadenomas. Hypertension 64 438-444.

Tunny, T.J., Gordon, R.D., Klemm, S.A., Cohn, D., 1991. Histological and biochemical distinctiveness of atypical aldosterone-producing adenomas responsive to upright posture and angiotensin. Clin. Endocrinol. (Oxf) 34, 363-369.

Williams, T.A., Monticone, S., Schack, V.R., Stindl, J., Burrello, J., Buffolo, F., et al., 2014 Somatic ATP1A1, ATP2B3, and KCNJ5 mutations in aldosterone-producing adenomas. Hypertension 63, 188-195.

Young, W.F., Stanson, A.W., Thompson, G.B., Grant, C.S., Farley, D.R., van Heerden, J.A., 2004. Role for adrenal venous sampling in primary aldosteronism. Surgery 136, 1227-1235. 